\title{
EVERY THREE-SPHERE OF POSITIVE RICCI CURVATURE CONTAINS A MINIMAL EMBEDDED TORUS
}

\author{
BRIAN WHITE
}

One of the most celebrated theorems of differential geometry is the 1929 theorem of Lusternik and Schnirelmann, which states that for every riemannian metric on the 2-sphere there exist at least three simple closed geodesics. Jurgen Jost $[\mathbf{J}]$ (following important work of Pitts [P] and Simon and Smith [SS]) has recently generalized this result by showing that for every riemannian metric on $S^{3}$, there exist at least 4 minimal embedded 2-spheres. This is optimal in that there are metrics for which the number of embedded minimal 2-spheres is exactly four [W2,4.5]. However, one can also ask about surfaces of higher genus, and here our knowledge is very incomplete. On the one hand, Lawson [L] showed that $S^{3}$ with its standard metric contains embedded minimal surfaces of every orientable toplogical type, and recently Pitts and Rubinstein [PR] have discovered many new infinite families of examples. But for general metrics on $S^{3}$, no known theorem asserts the existence of any minimal surface other than a sphere. The present paper takes a first step in this direction by proving:

THEOREM 3. For every $C^{4}$ riemannian metric $\gamma$ of positive ricci curvature on $S^{3}$, there exists at least one minimal embedded torus.

I conjecture that every metric on $S^{3}$ admits at least 5 minimal embedded tori, but I can prove it (by a perturbation argument [W2,4.4]) only for metrics that are close to the standard metric.

Peliminaries. The proof uses the following facts about the space of all minimal surfaces for varying riemannian metrics. The facts are proved in [W2] using the implicit function theorem. Let $N$ be a compact 3-manifold, $\Gamma$ be an open set of $C^{4}$ metrics on $N$, and $\mathcal{M}$ be the set of pairs $(\gamma, S)$ where $\gamma \in \Gamma$ and $S \subset N$ is a smooth embedded $\gamma$-minimal surface.

THEOREM 1 [W2, 2.1,2.2,5.1]. The set $\mathcal{M}$ is a smooth Banach manifold, and the map

$$
\begin{gathered}
\Pi: \mathcal{M} \rightarrow \Gamma \\
\Pi:(\gamma, S) \mapsto \gamma
\end{gathered}
$$

is a smooth map. Almost every (in the sense of Baire category) $\gamma$ is a regular value of $\Pi$, i.e., each element of $\Pi^{-1}(\gamma)$ is a nondegenerate critical point of the area functional.

Received by the editors October 26, 1988.

1980 Mathematics Subject Classification. Primary 58E12, 53A10, 49F10.

The author was partially funded by NSF grants DMS85-53231 (PYI) and DMS87-03537.

(C) 1989 American Mathematical Society $0273-0979 / 89 \$ 1.00+\$ .25$ per page 
Let $\mathcal{M}_{0}$ be the union of one or more connected components of $\mathcal{M}$. If $\Gamma$ is connected and if $\Pi: \mathcal{M}_{0} \rightarrow \Gamma$ is a proper map (inverse images of compact sets are compact), then $\Pi \mid \mathcal{M}_{0}$ has a mapping degree $d$ such that for each regular value $\gamma$,

$$
d=\sum_{(\gamma, S) \in \mathcal{M}_{0}}(-1)^{\operatorname{index}(S)}
$$

REMARK 1. If for some $\gamma, \Pi^{-1}(\gamma)=\varnothing$, then $\gamma$ is a regular value of $\Pi$ and so $d$ would have to be 0 by (1).

REMARK 2. This theorem remains true for simple immersed minimal surfaces. An immersion is simple if there is an $x \in N$ that is covered exactly once.

If $(\gamma, S) \in \mathcal{M}_{0}$ and $S$ has nullity 0 , then $S$ is a nondegenerate critical point of the area functional, and $(\gamma, S)$ is isolated in $\Pi^{-1}(\gamma)$ and contributes $(-1)^{\operatorname{index}(S)}$ to the degree. It sometimes happens that $\Pi^{-1}(\gamma)$ contains a compact $k$-dimensional manifold $\Sigma$ of surfaces. Of course the surfaces in $\Sigma$ are degenerate critical points, but if each of them has nullity equal to $k$, then $\Sigma$ is said to be a nondegenerate critical manifold and has nice properties.

THEOREM 2 [W2,5.1]. Suppose $\Pi^{-1}\left(\gamma_{0}\right)$ contains a nondegenerate critical manifold $\Sigma$. Then there is a neighborhood $\Gamma_{0} \subset \Gamma$ of $\gamma_{0}$ and a connected component $\mathcal{M}_{0}$ of $\Pi^{-1}\left(\Gamma_{0}\right)$ such that

$$
\Pi^{-1}\left(\gamma_{0}\right) \cap M_{0}=\Sigma
$$

and

$$
\operatorname{deg}\left(\Pi \mid \mathcal{M}_{0}\right)=\chi(\Sigma)(-1)^{\operatorname{index}(\Sigma)}
$$

where $\chi(\Sigma)$ is the euler characteristic of $\Sigma$ and index $(\Sigma)$ is equal to the index of $S$ for each $(\gamma, S) \in \Sigma$.

For example consider the clifford tori. (A clifford torus is the set of points in $S^{3} \subset R^{4}$ equidistant from a pair of orthogonal planes through the origin.) The set of all such tori (or, equivalently, the set of pairs of orthogonal planes in $R^{4}$ ) is topologically the 4-manifold $R P^{2} \times R P^{2}$. Straightforward calculations show that each clifford torus is a minimal surface with nullity 4 and index 1 . Thus the hypotheses of Theorem 2 are satisfied.

The Theorem.

THEOREM 3. For every $C^{4}$ metric $\gamma$ of positive ricci curvature on $S^{3}$, there exists at least one embedded torus that is minimal with respect to $\gamma$.

Proof. Let $\gamma_{0}$ be the standard metric on $S^{3}$ and let $S^{1}=\{z \in C:|z|=$ 1) act on $S^{3} \subset R^{4}=C^{2}$ by complex multiplication. Let $R_{n}: S^{3} \rightarrow S^{3}$ be multiplication by $e^{2 \pi i / n}$. We first prove the following:

LEMMA. There is an $n_{0}$ such that if $n \geq n_{0}$ and if $M \subset S^{3}$ is an embedded $\gamma_{0}$-minimal torus with $R_{n}(M)=M$, then $M$ is a clifford torus. 
Proof. Suppose not. Then there is a sequence $n(i) \rightarrow \infty$ of integers and a sequence $M_{i}$ of embedded minimal tori, none of which is a clifford torus, such that $R_{n(i)}\left(M_{i}\right)=M_{i}$.

By the compactness theorem of Choi and Schoen [CS], there is a convergent subsequence, which we may assume to be the original sequence: $M_{i} \rightarrow M$. Fix a real number $t>0$ and let $k(i)$ be the greatest integer less than or equal to $\operatorname{tn}(i)$, so that

$$
\frac{k(i)}{n(i)} \rightarrow t
$$

By hypothesis

$$
e^{\frac{2 \pi \imath}{n(1)}}\left(M_{i}\right)=M_{i}
$$

Thus

$$
e^{2 \pi i \frac{k(t)}{n(t)}}\left(M_{i}\right)=\left(e^{\frac{2 \pi i}{n(t)}}\right)^{k(i)}\left(M_{i}\right)=M_{i}
$$

so, letting $i \rightarrow \infty$,

$$
e^{2 \pi i t}(M)=M
$$

Since this holds for each $t, M$ is invariant under the $S^{1}$ action. Let

$$
h: S^{3} \subset C^{2} \rightarrow S^{2} \cong C \cup\{\infty\}
$$

be the Hopf map

$$
h(z, w)=z / w .
$$

In more geometrical terms, $h: S^{3} \subset C^{2} \rightarrow C P^{1} \cong S^{2}$ maps each point to the complex line that contains it. The $S^{1}$ invariance of $M$ means that there is a curve $T \subset S^{2}$ such that

$$
M=h^{-1}(T) .
$$

The area of the inverse image (under $h$ ) of a curve is a constant times the length of the curve. Thus the minimality of $M$ implies that $T$ is a union of geodesics. Since $M$ is embedded, $T$ is a single great circle and so $M$ is a clifford torus. It follows from Theorem 2 (and the example given after it) that for sufficiently large $i, M_{i}$ is a clifford torus.

Now to prove the theorem, let $\Gamma$ be the set of $C^{4}$ metrics of positive ricci curvature on $S^{3}, \mathcal{M}$ be the Banach manifold of Theorem 1 , and $\mathcal{M}_{0}$ be the set of $(\gamma, S) \in \mathcal{M}$ such that $S$ is a torus. The space $\Gamma$ is connected by a theorem of Hamilton [H]. By the compactness theorem of Choi and Schoen [CS], $\Pi \mid \mathcal{M}_{0}$ is proper and therefore (by Theorem 1) has a mapping degree; we will show that the degree is not zero. Let $p$ be a prime number greater than the $n_{0}$ of the lemma. Let $\gamma_{0}$ be the standard metric on $S^{3}$ and let $\Gamma_{0} \subset \Gamma$ be a neighborhood of $\gamma_{0}$ such that $\Pi^{-1}\left(\Gamma_{0}\right)$ contains a connected component $\mathcal{M}_{\text {cliff }}$ such that

$$
\Pi^{-1}\left(\gamma_{0}\right) \cap \mathcal{M}_{\text {cliff }}
$$

is precisely the set of clifford tori. (This is possible by Theorem 2.) Let

$$
\mathcal{M}_{\text {other }}=\Pi^{-1}\left(\Gamma_{0}\right) \cap \mathcal{M}_{0} \backslash \mathcal{M}_{\text {cliff }} \text {. }
$$


By the lemma we may choose $\Gamma_{0}$ small enough that if $(\gamma, S) \in \mathcal{M}_{\text {other }}$ then $R_{p}(S) \neq S$. Note that since $p$ is prime, if $T \subset S^{3}$ is an embedded submanifold that is not $R_{p}$ invariant, then there is an $x \in T$ such that $x \notin\left(R_{p}\right)^{k}(T)$ for $1 \leq k<p$. That is, $T$ becomes a simply immersed submanifold in the quotient space $S^{3} / R_{p}$. According to Theorem 1, for almost every metric $\gamma$ on $S^{3} / R_{p}$, each simple $\gamma$-minimal surface has nullity 0 . Equivalently, for almost every $R_{p}$ invariant metric $\gamma$ on $S^{3}$, each $\gamma$-minimal surface that is not $R_{p}$ invariant has nullity 0 . Fix any such $R_{p}$ invariant metric $\gamma \in \Gamma_{0}$.

Now if $(\gamma, S) \in \mathcal{M}_{\text {other }}$, then so is $\left(\gamma,\left(R_{p}\right)^{k} S\right)$ for $1 \leq k<p$. Furthermore, these $p$ surfaces are distinct and all have the same index (and nullity $0)$. Thus

$$
\begin{aligned}
\operatorname{deg}\left(\Pi \mid \mathcal{M}_{\text {other }}\right) & =\sum_{(\gamma, S) \in \mathcal{M}_{\text {other }}}(-1)^{\operatorname{index}(S)} \\
& \cong 0 \quad \bmod p .
\end{aligned}
$$

On the other hand, by Theorem 2 and the example following it:

$$
\begin{aligned}
\operatorname{deg}\left(\Pi \mid \mathcal{M}_{\text {cliff }}\right) & =\chi\left(R P^{2} \times R P^{2}\right)(-1)^{1} \\
& =-1 .
\end{aligned}
$$

Thus

$$
\begin{aligned}
\operatorname{deg}\left(\Pi \mid \mathcal{M}_{0}\right) & =\operatorname{deg}\left(\Pi \mid \mathcal{M}_{\text {cliff }}\right)+\operatorname{deg}\left(\Pi \mid \mathcal{M}_{\text {other }}\right) \\
& \cong-1 \quad \bmod p .
\end{aligned}
$$

Since this holds for arbitrarily large $p$, in fact $\operatorname{deg}\left(\Pi \mid \mathcal{M}_{0}\right)$ must be -1 . The theorem follows immediately (see Remark 1 after Theorem 1).

REMARK 1. The argument above for $\mathcal{M}_{\text {other }}$ also shows

THEOREM 4. Let $\mathcal{M}_{g}$ be the Banach manifold of pairs $(\gamma, S)$ where $\gamma$ is a $C^{4}$ metric of positive ricci curvature on $S^{3}$ and $S \subset S^{3}$ is an embedded surface of genus $g+1$ that is minimal with respect to $\gamma$. Then

$$
\operatorname{deg}\left(\Pi \mid \mathcal{M}_{g}\right)=0
$$

unless $g=0$.

REMARK 2. Mapping degrees were first applied to minimal surface theory by Tomi and Tromba [TT]. Additional applications were given in [W1]. Theorem 3 is the first result for which it is necessary to use the integral mapping degree rather than the simpler mod 2 degree.

\section{REFERENCES}

[CS] H. I. Choi and R. Schoen, The space of minimal embeddings of a surface into a three dimensional manifold of positive ricci curvature, Invent. Math. 81 (1985), 387-394.

[H] R. Hamilton, Three-manifolds with positive ricci curvature, J. Differential Geom. 17 (1982), 255-306.

[J] J. Jost, Embedded minimal surfaces in manifolds diffeomorphic to the three dimensional ball or sphere, preprint.

[L] H. B. Lawson, Complete minimal surfaces in $S^{3}$, Ann. of Math. (2) 92 (1970), 335-374.

[P] J. Pitts, Existence and regularity of minimal surfaces on riemannian manifolds, Princeton Univ. Press, Princeton, N.J., 1981. 
[PR] J. Pitts and H. Rubinstein, Equivariant minimax and minimal surfaces in geometric 3-manifolds, Bull. Amer. Math. Soc. (N.S.) 19 (1988), 303-309.

[SS2] L. Simon and F. Smith, On the existence of embedded minimal two-spheres in the three-sphere, endowed with an arbitrary riemannian metric, preprint.

[TT] F. Tomi and A. J. Tromba, Extreme curves bound embedded minimal surfaces of the type of the disk, Math. Z. 158 (1978), 137-145.

[W1] B. White, New applications of mapping degrees to minimal surface theory, J. Differential Geom. 29 (1989), 143-152.

[W2] _ , The space of minimal submanifolds for varying riemannian metrics, preprint.

Department of Mathematics, Stanford University, Stanford, California 94305 
